\title{
Clustering Unclustered Data Unsupervised Binary Labeling of Two Datasets Having Different Class Balances
}

\author{
Marthinus Christoffel du Plessis and Masashi Sugiyama \\ Department of Computer Science, Tokyo Institute of Technology, \\ 2-12-1 O-okayama, Meguro-ku, Tokyo 152-8552, Japan, \\ christo@sg.cs.titech.ac.jp, sugi@cs.titech.ac.jp
}

\begin{abstract}
We consider the unsupervised learning problem of assigning labels to unlabeled data. A naive approach is to use clustering methods, but this works well only when data is properly clustered and each cluster corresponds to an underlying class. In this paper, we first show that this unsupervised labeling problem in balanced binary cases can be solved if two unlabeled datasets having different class balances are available. More specifically, estimation of the sign of the difference between probability densities of two unlabeled datasets gives the solution. We then introduce a new method to directly estimate the sign of the density difference without density estimation. Finally, we demonstrate the usefulness of the proposed method against several clustering methods on various toy problems and real-world datasets.
\end{abstract}

\section{Introduction}

Gathering labeled data is expensive and time consuming in many practical machine learning problems, and therefore class labels are often absent. In this paper, we consider the problem of labeling, which is aimed at giving a label to each sample. Labeling is similar to classification, but it is slightly simpler than classification because classes do not have to be specified. That is, labeling just tries to split unlabeled samples into disjoint subsets, and class labels such as male/female or positive/negative are not assigned to samples.

A naive approach to the labeling problem is to use a clustering technique which is aimed at assigning a label to each sample of the dataset to divide the dataset into disjoint clusters. The tacit assumption in clustering is that the clusters correspond to the underlying classes. However, this assumption is often violated in practical datasets, for example, when clusters are not well separated or a dataset exhibits within-class multimodality.

An example of the labeling problem is illustrated in Figure 1. Figure 1(a) denotes the densities of the two classes. Figure 1(b) denotes samples drawn from a mixture of the two original densities. Because the two clusters are highly overlapping, it may not be possible to properly label them by a clustering method.

In this paper we show that if one more dataset with a different class balance is available (Figure 1() ), the labeling problem can be solved (Figures 1(d) and 


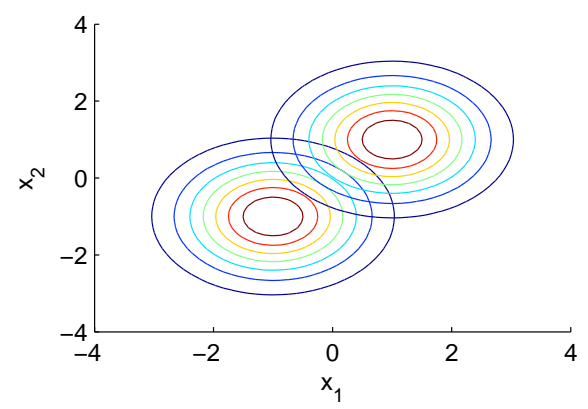

(a) Original distributions

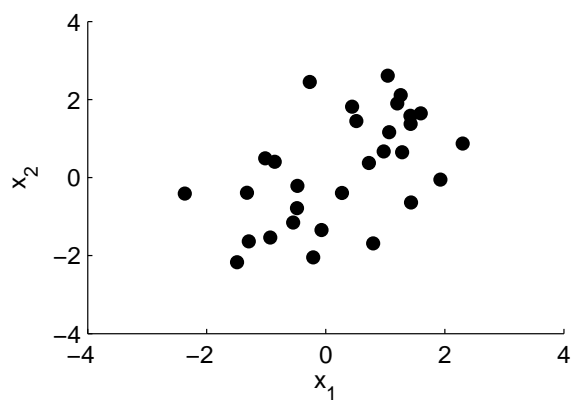

(b) Samples in $\mathcal{X}_{p}$

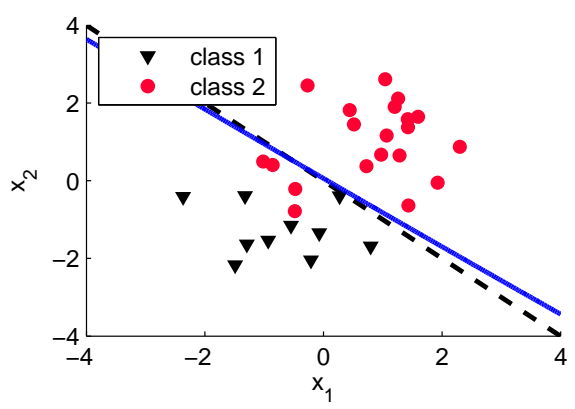

(d) Labeling of $\mathcal{X}_{p}$

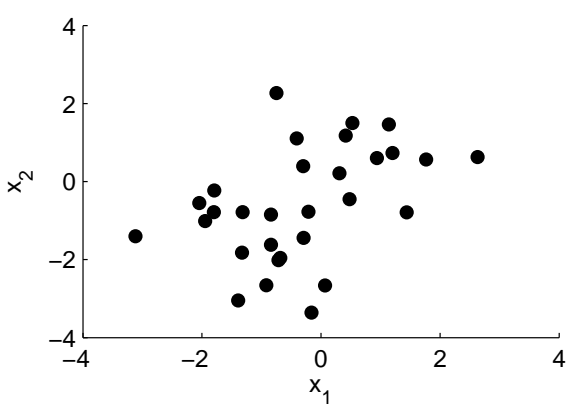

(c) Samples in $\mathcal{X}_{p^{\prime}}$

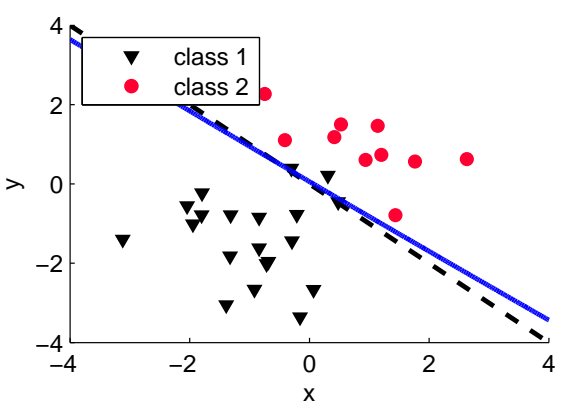

(e) Labeling of $\mathcal{X}_{p^{\prime}}$

Fig. 1. Illustrative example of labeling samples from unbalanced datasets. Figures $1 \mathrm{~b}$ and $1 \mathrm{c}$ show the samples of the two datasets which differ only by class balance (the datasets are denoted as $\mathcal{X}_{p}$ and $\mathcal{X}_{p^{\prime}}$ ). The discriminant estimated by the method that we propose in this paper is given in blue and the optimal discriminant is given in the black dashed line. The true underlying class labels (which are unknown) are illustrated in red and black.

T(或). More specifically, we show that a labeling for the samples can be obtained by estimating the sign of the difference between probability densities of two unlabeled datasets. A naive way is to first separately estimate two densities from two 
sets of samples and then take the sign of their difference to obtain a labeling. However, this naive procedure violates Vapnik's principle 11:

If you possess a restricted amount of information for solving some problem, try to solve the problem directly and never solve a more general problem as an intermediate step. It is possible that the available information is sufficient for a direct solution but is insufficient for solving a more general intermediate problem.

This principle was used in the development of support vector machines (SVMs): Rather than modeling two classes of samples, SVM directly learns a decision boundary that is sufficient for performing pattern recognition.

In the current context, estimating two densities is more general than labeling samples. Thus, the above naive scheme may be improved by estimating the density difference directly and then taking its sign to obtain the class labels. Recently, a method was introduced to directly estimate the density difference, called the least-squares density difference (LSDD) estimator [2. Thus, the use of LSDD for labeling is expected to improve the performance.

However, the LSDD-based procedure is still indirect; directly estimating the sign of the density difference would be the most suitable approach to labeling. In this paper, we show that the sign of the density difference can be directly estimated by lower-bounding the $L_{1}$-distance between probability densities. Based on this, we give a practical algorithm for labeling and illustrate its usefulness through experiments on various real-world datasets.

\section{Problem Formulation and Fundamental Approaches}

In this section, we formulate the problem of labeling, give our fundamental strategy, and consider two naive approaches.

\subsection{Problem Formulation}

Suppose that there are two probability distributions $p(\boldsymbol{x}, y)$ and $p^{\prime}(\boldsymbol{x}, y)$ on $\boldsymbol{x} \in$ $\mathbb{R}^{d}$ and $y \in\{1,-1\}$, which are different only in class balances:

$$
p(y) \neq p^{\prime}(y) \quad \text { but } \quad p(\boldsymbol{x} \mid y)=p^{\prime}(\boldsymbol{x} \mid y) .
$$

From these distributions, we are given two sets of unlabeled samples:

$$
\mathcal{X}_{p}=\left\{\boldsymbol{x}_{i}\right\}_{i=1}^{n} \stackrel{\text { i.i.d. }}{\sim} p(\boldsymbol{x}) \text { and } \mathcal{X}_{p^{\prime}}=\left\{\boldsymbol{x}_{j}^{\prime}\right\}_{j=1}^{n^{\prime}} \stackrel{\text { i.i.d. }}{\sim} p^{\prime}(\boldsymbol{x}) .
$$

The goal of labeling is to obtain a labeling for the two sets of samples, $\mathcal{X}_{p}$ and $\mathcal{X}_{p^{\prime}}$, that corresponds to the underlying class labels $\left\{y_{i}\right\}_{i=1}^{n}$ and $\left\{y_{j}^{\prime}\right\}_{j=1}^{n^{\prime}}$. However, different from classification, we do not obtain correct class labels, but we obtain correct class separation up to label commutation. 


\section{$2.2 \quad$ Fundamental strategy}

We wish to obtain a labeling for samples in $\mathcal{X}_{p}$ and $\mathcal{X}_{p^{\prime}}$. Here we show that we can obtain the solution for the case where the class priors are equal. We may write the class-posterior distribution for the equal prior case as

$$
q(y=1 \mid \boldsymbol{x})=\frac{p(\boldsymbol{x} \mid y) q(y)}{q(\boldsymbol{x})},
$$

where $q(y=1)=q(y=-1)=\frac{1}{2}$. A class label can then be assigned to a point by evaluating

$$
\operatorname{sign}[q(y=1 \mid \boldsymbol{x})-q(y=-1 \mid \boldsymbol{x})]
$$

We can write the criterion as

$$
\begin{aligned}
q(y=1 \mid \boldsymbol{x})-q(y=-1 \mid \boldsymbol{x}) & =\frac{p(\boldsymbol{x} \mid y=1) \frac{1}{2}}{q(\boldsymbol{x})}-\frac{p(\boldsymbol{x} \mid y=-1) \frac{1}{2}}{q(\boldsymbol{x})} \\
& \propto p(\boldsymbol{x} \mid y=1)-p(\boldsymbol{x} \mid y=-1) .
\end{aligned}
$$

We do not have any labeled samples to calculate $p(\boldsymbol{x} \mid y=1)-p(\boldsymbol{x} \mid y=-1)$, but we can rewrite it in terms of marginal distributions. To see this, the above is multiplied with $p(y=1)-p^{\prime}(y=1)$, which gives

$$
\begin{aligned}
p(\boldsymbol{x} \mid y=1)-p(\boldsymbol{x} \mid y=-1) \propto & {\left[p(y=1)-p^{\prime}(y=1)\right][p(\boldsymbol{x} \mid y=1)-p(\boldsymbol{x} \mid y=-1)] } \\
\propto & p(\boldsymbol{x}, y=1)-p^{\prime}(\boldsymbol{x}, y=1) \\
& -p(y=1) p(\boldsymbol{x} \mid y=-1)+p^{\prime}(y=1) p(\boldsymbol{x} \mid y=-1) .
\end{aligned}
$$

Note that the sign may change since $p(y=1)-p^{\prime}(y=1)$ may be positive or negative. To write the third and fourth term as a joint distribution, we add and subtract $p(\boldsymbol{x} \mid y=-1)$, giving

$$
\begin{aligned}
p(\boldsymbol{x} \mid y=1)-p(\boldsymbol{x} \mid y=-1) & \propto p(\boldsymbol{x}, y=1)-p^{\prime}(\boldsymbol{x}, y=-1)+[1-p(y=1)] p(\boldsymbol{x} \mid y=-1) \\
& -\left[1-p^{\prime}(y=1)\right] p(\boldsymbol{x} \mid y=-1) .
\end{aligned}
$$

Since $p(y=-1)=1-p(y=1)$ and $p^{\prime}(y=-1)=1-p^{\prime}(y=1)$, we can express the above as

$$
q(y=1 \mid \boldsymbol{x})-q(y=-1 \mid \boldsymbol{x}) \propto p(\boldsymbol{x})-p^{\prime}(\boldsymbol{x}) .
$$

The exact class labels can not be recovered since the term $p(y=1)-p^{\prime}(y=1)$ can be positive or negative. Therefore, we assign the label $y \in\{1,-1\}$ to a point $\boldsymbol{x}$ according to the following criterion:

$$
y=\operatorname{sign}\left[p(\boldsymbol{x})-p^{\prime}(\boldsymbol{x})\right] .
$$

Thus, now we need a good method to estimate sign $\left[p(\boldsymbol{x})-p^{\prime}(\boldsymbol{x})\right]$. 


\subsection{Kernel Density Estimation}

A naive approach to estimating the sign of density-difference is to use kernel density estimators (KDEs) [3]. For Gaussian kernels, the KDE solutions are given by

$$
\widehat{p}(\boldsymbol{x}) \propto \sum_{i=1}^{n} \exp \left(-\frac{\left\|\boldsymbol{x}-\boldsymbol{x}_{i}\right\|^{2}}{2 \sigma^{2}}\right) \text { and } \widehat{p}^{\prime}(\boldsymbol{x}) \propto \sum_{j=1}^{n^{\prime}} \exp \left(-\frac{\left\|\boldsymbol{x}-\boldsymbol{x}_{j}^{\prime}\right\|^{2}}{2 \sigma^{\prime 2}}\right) .
$$

The Gaussian widths $\sigma$ and $\sigma^{\prime}$ may be determined based on least-squares crossvalidation [4. Finally, a labeling is obtained as

$$
y=\operatorname{sign}\left[\widehat{p}(\boldsymbol{x})-\widehat{p}^{\prime}(\boldsymbol{x})\right] .
$$

\subsection{Direct Estimation of the Density Difference}

KDE is a nice density estimator, but it is not necessarily suitable in densitydifference estimation, because small estimation error incurred in each density estimate can cause a big error in the final density-difference estimate. More intuitively, good density estimators tend to be smooth and thus a densitydifference estimator obtained from such smooth density estimators tends to be over-smoothed [5]6].

The density difference can be estimated in a single shot using the least-squares density difference (LSDD) approach [2]. In this approach, we directly fit a model $g(\boldsymbol{x})$ to the density difference under the square loss:

$$
\widehat{g}=\underset{g}{\operatorname{argmin}} \frac{1}{2} \int\left(g(\boldsymbol{x})-\left(p(\boldsymbol{x})-p^{\prime}(\boldsymbol{x})\right)\right)^{2} \mathrm{~d} \boldsymbol{x},
$$

which can be efficiently obtained for a kernel density-difference model. A comprehensive review of LSDD is provided in Appendix B. Finally, a labeling is obtained as

$$
y=\operatorname{sign}[\widehat{g}(\boldsymbol{x})] .
$$

\section{Direct Estimation of the Sign of the Density Difference}

We expect that an improved solution can be obtained by LSDD over KDEs due to more direct nature of LSDD. However, LSDD is still indirect because the sign of density difference is inspected after the density difference is estimated. In this section, we show how to directly estimate the sign of the density difference.

\subsection{Derivation of the Objective Function}

By lower-bounding the $L_{1}$-distance between probability densities, defined as

$$
\int\left|p(\boldsymbol{x})-p^{\prime}(\boldsymbol{x})\right| \mathrm{d} \boldsymbol{x}
$$


we can obtain the sign of the density difference. We begin by considering the following self-evident relation:

$$
|t| \geq t z, \text { if }|z| \leq 1 .
$$

We can apply this relation at each point $\boldsymbol{x}$, to obtain

$$
\left|p(\boldsymbol{x})-p^{\prime}(\boldsymbol{x})\right| \geq g(\boldsymbol{x})\left[p(\boldsymbol{x})-p^{\prime}(\boldsymbol{x})\right] \text { if }|g(\boldsymbol{x})| \leq 1, \forall \boldsymbol{x} .
$$

By applying the above inequality to Eq.(4) and maximizing with respect to $g(\boldsymbol{x})$, we can obtain the tightest lower bound as

$$
\begin{gathered}
\int\left|p(\boldsymbol{x})-p^{\prime}(\boldsymbol{x})\right| \mathrm{d} \boldsymbol{x} \geq \sup _{g} \int g(\boldsymbol{x})\left[p(\boldsymbol{x})-p^{\prime}(\boldsymbol{x})\right] \mathrm{d} \boldsymbol{x} \\
\text { s.t. }|g(\boldsymbol{x})| \leq 1, \quad \forall \boldsymbol{x} .
\end{gathered}
$$

It is straightforward to verify that the above relation will be met with equality when

$$
g(\boldsymbol{x})=\operatorname{sign}\left(p(\boldsymbol{x})-p^{\prime}(\boldsymbol{x})\right) .
$$

What makes the expression in the right-hand side of Eq.(5) especially useful is that the probability densities occur linearly in the integral. By replacing the integrals with sample averages and searching $g(\boldsymbol{x})$ from a parametric family (denoted as $g_{\boldsymbol{\alpha}}(\boldsymbol{x})$ ), we can write the above as

$$
\begin{aligned}
& \boldsymbol{\alpha}=\underset{\boldsymbol{\alpha}}{\arg \min } \frac{1}{n^{\prime}} \sum_{j=1}^{n^{\prime}} g_{\boldsymbol{\alpha}}\left(\boldsymbol{x}_{j}^{\prime}\right)-\frac{1}{n} \sum_{i=1}^{n} g_{\boldsymbol{\alpha}}\left(\boldsymbol{x}_{i}\right) \\
& \text { s.t. }\left|g_{\boldsymbol{\alpha}}(\boldsymbol{x})\right| \leq 1, \forall \boldsymbol{x} \text {. }
\end{aligned}
$$

\subsection{Optimization}

Here we briefly discuss how to solve the problem in Eq. (6). A more detailed explanation is given in Appendix A

The function in Eq. (6) should satisfy the constraint $|g(\boldsymbol{x})| \leq 1, \forall \boldsymbol{x}$. We can consider a clipped version of the function that always satisfies the constraint,

$$
\widetilde{g}(\boldsymbol{x})=R(g(\boldsymbol{x})), \quad \text { where } R(z)= \begin{cases}1 & z>1 \\ -1 & z<-1 \\ z & \text { otherwise }\end{cases}
$$

We use a linear-in-parameter model,

$$
g(\boldsymbol{x})=\sum_{\ell=1}^{b} \alpha_{\ell} \varphi_{\ell}(\boldsymbol{x}),
$$


where $\varphi_{\ell}(\boldsymbol{x})$ are the basis functions. Using the above definitions, we can rewrite Eq.(6) as

$$
J(\boldsymbol{\alpha})=\frac{1}{n^{\prime}} \sum_{i=1}^{n^{\prime}} R\left(\sum_{\ell=1}^{b} \alpha_{\ell} \varphi_{\ell}\left(\boldsymbol{x}_{i}^{\prime}\right)\right)-\frac{1}{n} \sum_{j=1}^{n} R\left(\sum_{\ell=1}^{b} \alpha_{\ell} \varphi_{\ell}\left(\boldsymbol{x}_{j}\right)\right)+\frac{\lambda}{2} \sum_{\ell=1}^{b} \alpha_{\ell}^{2},
$$

where $\frac{\lambda}{2} \sum_{\ell=1}^{b} \alpha_{\ell}^{2}$ is a regularization term. Although the above is a non-convex problem, we can efficiently find a local optimal solution using the convex-concave procedure (CCCP) [7. (also known as difference of convex (d.c.) programming [8]). The CCCP procedure requires the objective function to be split into a convex and concave part,

$$
J(\boldsymbol{\alpha})=J_{\mathrm{vex}}(\boldsymbol{\alpha})+J_{\text {cave }}(\boldsymbol{\alpha}) .
$$

The concave part is then upper-bounded as

$$
J_{\text {cave }}(\boldsymbol{\alpha}) \leq \bar{J}_{\text {cave }}(\boldsymbol{\alpha}, \boldsymbol{b}, \boldsymbol{c}),
$$

where the bound is specified by $\boldsymbol{b}$ and $\boldsymbol{c}$ (details are given in Appendix $\mathrm{A}$ ). This bound is convex w.r.t. $\boldsymbol{b}$ and $\boldsymbol{c}$ if $\boldsymbol{\alpha}$ is fixed. Using this bound, the optimization problem can then be expressed as

$$
J(\boldsymbol{\alpha}) \leq J_{\mathrm{vex}}(\boldsymbol{\alpha})+\bar{J}_{\text {cave }}(\boldsymbol{\alpha}, \boldsymbol{b}, \boldsymbol{c}) .
$$

The strategy to minimize $J(\boldsymbol{\alpha})$ is then to alternately minimize the right-hand side by minimizing w.r.t. $\boldsymbol{\alpha}$ (keeping $\boldsymbol{b}$ and $\boldsymbol{c}$ constant) and minimize w.r.t. $\boldsymbol{b}$ and $\boldsymbol{c}$ (keeping $\boldsymbol{\alpha}$ constant). Minimization w.r.t. $\boldsymbol{\alpha}$ minimizes the current upper bound and minimization w.r.t. $\boldsymbol{b}$ and $\boldsymbol{c}$ corresponds to tightening the bound at the current point.

Minimization w.r.t. $\boldsymbol{b}$ and $\boldsymbol{c}$ can be performed by

$$
b_{i}=\left\{\begin{array}{ll}
0 & \sum_{\ell=1}^{b} \alpha_{\ell} \varphi_{\ell}\left(\boldsymbol{x}_{i}^{\prime}\right)<1, \\
1 & \text { otherwise }
\end{array} \quad \text { and } c_{j}= \begin{cases}0 & \sum_{\ell=1}^{b} \alpha_{\ell} \varphi\left(\boldsymbol{x}_{j}\right)<-1, \\
1 & \text { otherwise }\end{cases}\right.
$$

Minimization of the upper bound (assuming $\boldsymbol{b}$ and $\boldsymbol{c}$ is constant) can be performed by solving the following convex quadratic problem:

$$
\begin{gathered}
\bar{J}(\boldsymbol{\alpha})=\frac{1}{n^{\prime}} \sum_{i=1}^{n^{\prime}} \xi_{i}^{\prime}+\frac{1}{n} \sum_{j=1}^{n} \xi_{j}-\sum_{\ell=1}^{b} \alpha_{\ell}\left(\frac{1}{n^{\prime}} \sum_{i=1}^{n^{\prime}} b_{i} \varphi_{\ell}\left(\boldsymbol{x}_{i}^{\prime}\right)+\frac{1}{n} \sum_{j=1}^{n} c_{j} \varphi_{\ell}\left(\boldsymbol{x}_{j}\right)\right)+\frac{\lambda}{2} \sum_{\ell=1}^{b} \alpha_{\ell}^{2} \\
\text { s.t. } \xi_{i}^{\prime} \geq 0, \xi_{i}^{\prime} \geq \sum_{\ell=1}^{b} \alpha_{\ell} \varphi_{\ell}\left(\boldsymbol{x}_{i}^{\prime}\right)+1, \forall i=1, \ldots, n^{\prime} \\
\xi_{j} \geq 0, \xi_{j} \geq \sum_{\ell=1}^{b} \alpha_{\ell} \varphi_{\ell}\left(\boldsymbol{x}_{j}\right)-1 \forall j=1, \ldots, n .
\end{gathered}
$$

The above constrained problem can be solved with an off-the-shelf QP solver.

Our final optimization algorithm is summarized below: 
1. Initialize the starting value:

$$
\boldsymbol{\alpha}^{1} \leftarrow \underset{\boldsymbol{\alpha}}{\arg \min } J_{\mathrm{vex}}(\boldsymbol{\alpha})
$$

2. For $t=1, \ldots T$ :

(a) Tighten the upper-bound: Obtain $\boldsymbol{b}$ and $\boldsymbol{c}$ as

$$
\boldsymbol{b}, \boldsymbol{c} \leftarrow \underset{\boldsymbol{b}, \boldsymbol{c}}{\arg \min } \bar{J}\left(\boldsymbol{\alpha}^{t}, \boldsymbol{b}, \boldsymbol{c}\right),
$$

by using Eq.(8).

(b) Minimize the upper bound:

Set

$$
\boldsymbol{\alpha}^{t+1} \leftarrow \underset{\boldsymbol{\alpha}}{\arg \min } J_{\mathrm{vex}}(\boldsymbol{\alpha})+\bar{J}_{\text {cave }}(\boldsymbol{\alpha}, \boldsymbol{b}, \boldsymbol{c},)
$$

by solving the convex problem in Eq.(9).

In practice, Gaussian kernels centered at the sample points in $\mathcal{X}_{p}$ and $\mathcal{X}_{p^{\prime}}$ are chosen as the basis functions. All hyper-parameters are set by cross-validation.

\section{Experiments}

We first illustrate the operation of our method and characterize the failures of other methods on various toy examples. Then we use real-world benchmark data to show the superiority of our algorithm.

\subsection{Numerical Illustration}

Toy Problem 1: We illustrate the problem and our method with a simple example. Suppose that the class-conditional densities for the two classes are given as

$$
p(\boldsymbol{x} \mid y=1)=\mathcal{N}_{\boldsymbol{x}}\left(-\mathbf{1}_{2}, \boldsymbol{I}_{2 \times 2}\right) \text { and } p(\boldsymbol{x} \mid y=-1)=\mathcal{N}_{\boldsymbol{x}}\left(\mathbf{1}_{2}, \boldsymbol{I}_{2 \times 2}\right),
$$

where $\mathcal{N}_{\boldsymbol{x}}(\boldsymbol{\mu}, \boldsymbol{\Sigma})$ denotes the normal density with mean $\boldsymbol{\mu}$ and covariance $\boldsymbol{\Sigma}$ w.r.t. $\boldsymbol{x} . \boldsymbol{1}_{2}$ is a $2 \times 1$ vector of ones and $\boldsymbol{I}$ is a $2 \times 2$ identity matrix. We generate 2 sets of 30 samples with class-priors $p(y=1)=0.3$ and $p^{\prime}(y=1)=0.7$, respectively. The result is illustrated in Figure 1. As can be seen from this example, we are able to obtain a labeling of the classes that roughly corresponds to the true (unknown) labels of the data. 
Toy Problem 2: One way to obtain a labeling is to use clustering. The tacit assumption in clustering is that samples in the same cluster belong the same class. This assumption however is not always be true, for example, when the class conditional densities are multimodal. Here we consider a problem with the following class conditional densities:

$$
\begin{gathered}
p(\boldsymbol{x} \mid y=1)=\frac{1}{2} \mathcal{N}_{\boldsymbol{x}}\left(\left[\begin{array}{ll}
3 & 0
\end{array}\right]^{\top}, \boldsymbol{I}_{2 \times 2}\right)+\frac{1}{2} \mathcal{N}_{\boldsymbol{x}}\left(\left[\begin{array}{ll}
-3 & 0
\end{array}\right]^{\top}, \boldsymbol{I}_{2 \times 2}\right) \\
p(\boldsymbol{x} \mid y=-1)=\frac{1}{2} \mathcal{N}_{\boldsymbol{x}}\left(\left[\begin{array}{ll}
0 & 3
\end{array}\right]^{\top}, \boldsymbol{I}_{2 \times 2}\right)+\frac{1}{2} \mathcal{N}_{\boldsymbol{x}}\left([0-3]^{\top}, \boldsymbol{I}_{2 \times 2}\right) .
\end{gathered}
$$

The two distributions are plotted in Figure 2a, We can try to obtain a class label by performing clustering on $\mathcal{X}_{p} \cup \mathcal{X}_{p^{\prime}}, 1$. The results for k-means and spectral clustering, given in Figures $2 \mathrm{~d}$ and $2 \mathrm{e}$, show that these methods fail to reveal the true labeling. On the other hand, the proposed method still gives a reasonable result (Figure 2ff).

\subsection{Benchmark Datasets}

We compare our method against several competing methods on benchmark datasets. For each experiment, we constructed the datasets $\mathcal{X}_{p}$ and $\mathcal{X}_{p^{\prime}}$ by drawing $n$ and $n^{\prime}$ samples from the positive and negative classes of the datasets according to a prior of $p(y=1)$ and $p^{\prime}(y=1)$. The labeling was then performed using these two datasets. Since we can obtain a labeling, but cannot determine the original class labels, we cannot measure the performance using the misclassification rate directly. Assume that the label assigned for the sample $\boldsymbol{x}_{i}$ is

$$
l_{i}= \begin{cases}-1 & p\left(\boldsymbol{x}_{i}\right)-q\left(\boldsymbol{x}_{i}\right)<0 \\ 1 & \text { otherwise }\end{cases}
$$

The misclassification rate (MCR) assuming that the current labels are correct is

$$
\mathrm{MCR}:=\frac{1}{n} \sum_{i: l_{j} \neq y_{i}} 1+\frac{1}{n^{\prime}} \sum_{j: l_{j}^{\prime} \neq y_{i}^{\prime}} 1 .
$$

The misclassification rate assuming that the labels are the opposite is $1-$ MCR. We define the labeling error rate (LER) as

$$
\mathrm{LER}:=\min (\mathrm{MCR}, 1-\mathrm{MCR}) .
$$

Note that this definition is somewhat more optimistic than using the misclassification rate. The smaller the dataset is, the lower the error would be for randomly

\footnotetext{
1 If clustering is performed separately on $\mathcal{X}_{p}$ and $\mathcal{X}_{p^{\prime}}$, we do not know which clusters in each dataset correspond to the clusters in the other dataset. We can also not perform clustering on one dataset and apply it to the other dataset, since most clustering methods do not give out of sample labeling. For these reasons, it makes most sense to perform clustering on the combined dataset.
} 


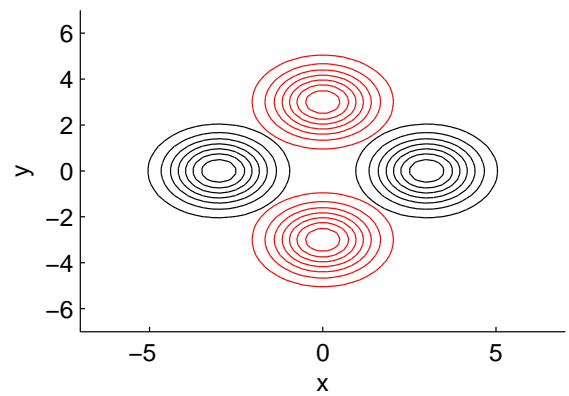

(a) Original distributions

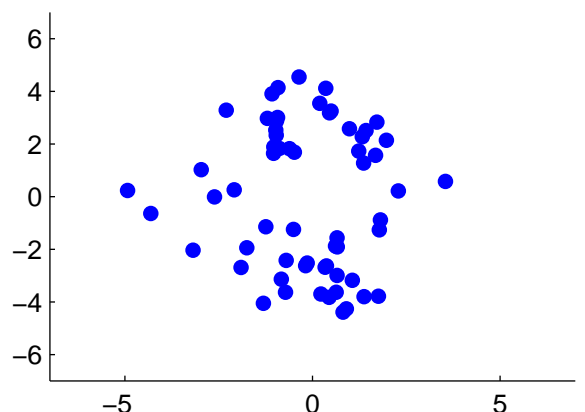

(b) $\mathcal{X}_{p}$

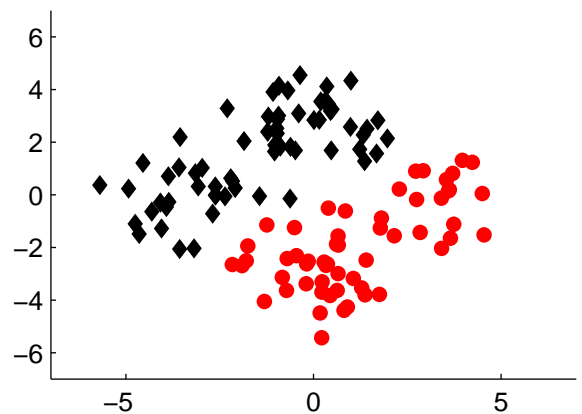

(d) K-means

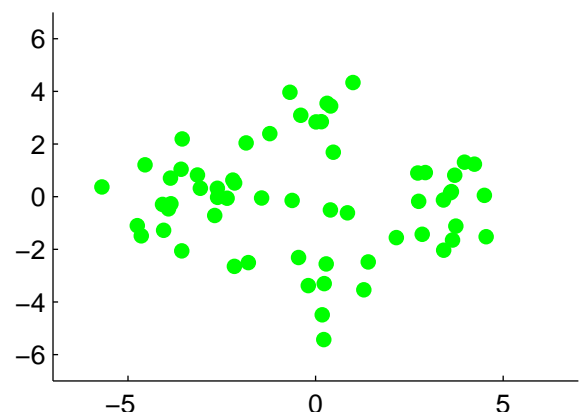

(c) $\mathcal{X}_{p^{\prime}}$

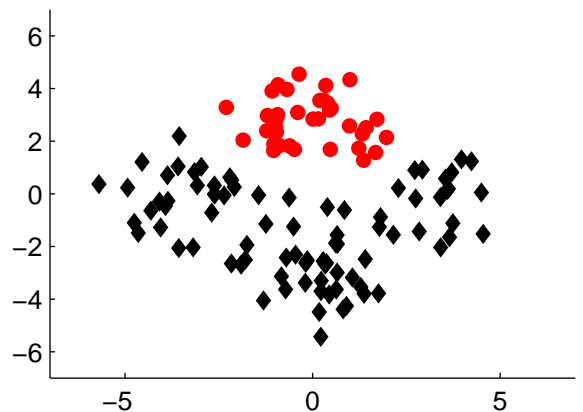

(e) Spectral clustering

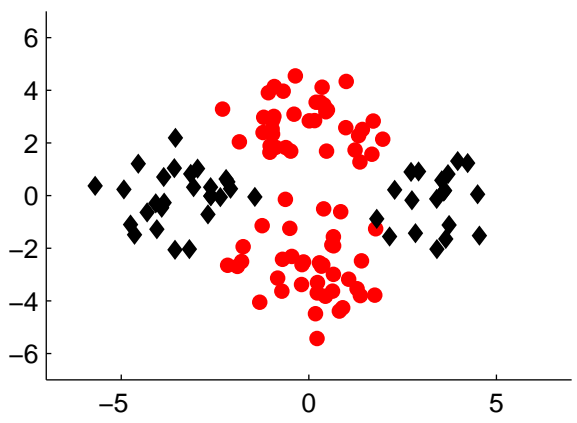

(f) Direct estimation

Fig. 2. Illustration of within-class multimodality and clustering. 
assigning labels to samples: The expected LER for randomly assigning labels to samples (with equal probability) is

$$
\frac{1}{2^{n+n^{\prime}}\left(n+n^{\prime}\right)} \sum_{i=0}^{n+n^{\prime}} \min \left(i, n+n^{\prime}-i\right)\left(\begin{array}{c}
n+n^{\prime} \\
i
\end{array}\right) .
$$

For $n+n^{\prime}=40,60,80$, the expected labeling error rate is $0.437,0.449,0.456$.

We compared the following methods:

- Direct Sign Density Difference (DSDD) Estimation (proposed): Directly estimate sign $\left(p(\boldsymbol{x})-p^{\prime}(\boldsymbol{x})\right)$ using the method described in Section 3 . Hyperparameters are selected via cross validation.

- Least-Squares Density Difference (LSDD) Estimation: Estimate sign $\left[p(\boldsymbol{x})-p^{\prime}(\boldsymbol{x})\right]$ by estimating $p(\boldsymbol{x})-p^{\prime}(\boldsymbol{x})$ using the least squares fitting method [9]. Hyperparameters are selected via cross validation.

- Kernel Density Estimation (KDE): Estimate sign $\left[p(\boldsymbol{x})-p^{\prime}(\boldsymbol{x})\right]$ by estimating the densities $p(\boldsymbol{x})$ and $p^{\prime}(\boldsymbol{x})$ with kernel density estimation (KDE). Hyperparameters are selected using least-squares cross validation.

- K-Means (KM): Cluster the data into two clusters using the K-means algorithm.

- Spectral Clustering (SC): Cluster the data into two clusters using the spectral clustering algorithm [10]. The affinity matrix was constructed with 7 nearest neighbors.

- Squared-loss Mutual Information based Clustering (SMIC) : Cluster the data according to the SMIC method [11. SMIC was chosen since it provides model selection, avoiding the need for subjective parameter tuning.

We compare the performance of the methods by varying the class balance. Two class balances were selected: one with a large difference between the classes $\left(p(y=1)=0.2\right.$ and $\left.p^{\prime}(y=1)=0.8\right)$ and one with a small difference between the two priors $\left(p(y=1)=0.35\right.$ and $\left.p^{\prime}(y=1)=0.65\right)$. The average labeling error rate and standard deviation of the two experiments, with $\left|\mathcal{X}_{p}\right|=\left|\mathcal{X}_{p^{\prime}}\right|=40$ is given in Tables 1 and 2

From the results we see that methods which follow the approach proposed in Section 2 of estimating the sign of the density difference (i.e., DSDD, LSDD, and $\mathrm{KDE}$ ) generally work better than methods using the cluster structure of the data (i.e., KM, SC and SMIC). The thyroid dataset lends itself to interpretation of why these methods work better. The labels in the thyroid dataset correspond to healthy and diseased. The diseased label is caused by either a hyper-functioning or hypo-functioning thyroid. These two underlying causes cause within-class multimodality which may cause clustering-based methods to fail.

Among the methods which estimate the sign of the density difference, we see that DSDD generally performs better than LSDD and LSDD in turn performs better than KDE. This is as expected since KDE solves a more general problem than LSDD, and LSDD solves a more general problem than DSDD. This pattern is even more pronounced on the more difficult case where the class balances are close to each other (Table 21). 
Table 1. Labeling error rate for experiments with a class prior of $p(y=1)=0.2$ and $p^{\prime}(y=1)=0.8$. The size of each dataset was $\left|\mathcal{X}_{p}\right|=40$ and $\left|\mathcal{X}_{p^{\prime}}\right|=40$. The best method in terms of the mean error and comparable methods according to the twosided paired t-test at the significance level $5 \%$ are specified by bold face. The standard deviation of the labeling error rate is given in brackets.

\begin{tabular}{l|cccccc}
\hline Dataset & DSDD & LSDD & KDE & KM & SC & SMIC \\
\hline australian & $\mathbf{. 1 4 2}(.045)$ & $.174(.110)$ & $.211(.126)$ & $.266(.147)$ & $.381(.033)$ & $.303(.103)$ \\
banana & $.179(.097)$ & $\mathbf{. 1 7 0}(.070)$ & $.237(.147)$ & $.431(.068)$ & $.427(.141)$ & $.424(.141)$ \\
diabetes & $.246(.122)$ & $\mathbf{. 2 2 3}(.079)$ & $\mathbf{. 2 2 6}(.051)$ & $.372(.080)$ & $.380(.094)$ & $.370(.131)$ \\
german & $.268(.059)$ & $.281(.127)$ & $\mathbf{. 2 1 1}(.051)$ & $.437(.114)$ & $.448(.128)$ & $.439(.052)$ \\
heart & $\mathbf{. 1 7 6}(.051)$ & $\mathbf{. 1 7 4}(.047)$ & $.211(.074)$ & $.261(.131)$ & $.310(.032)$ & $.327(.107)$ \\
image & $\mathbf{. 1 9 8}(.078)$ & $.206(.047)$ & $\mathbf{. 2 0 1}(.049)$ & $.385(.093)$ & $.351(.119)$ & $.384(.135)$ \\
ionosphere & $\mathbf{. 1 5 7}(.059)$ & $.184(.106)$ & $.194(.123)$ & $.329(.145)$ & $.319(.113)$ & $.311(.174)$ \\
saheart & $.310(.093)$ & $\mathbf{. 2 0 5}(.048)$ & $.238(.113)$ & $.422(.121)$ & $.395(.113)$ & $.384(.072)$ \\
thyroid & $\mathbf{. 1 0 2}(.052)$ & $.121(.116)$ & $.207(.074)$ & $.328(.113)$ & $.326(.109)$ & $.305(.074)$ \\
twonorm & $.044(.085)$ & $.051(.072)$ & $.200(.028)$ & $\mathbf{. 0 3 6}(.054)$ & $.043(.069)$ & $.048(.071)$ \\
\hline
\end{tabular}

Table 2. Labeling error rate for experiments with a class prior of $p(y=1)=0.35$ and $p^{\prime}(y=1)=0.65$. The size of each dataset was $\left|\mathcal{X}_{p}\right|=40$ and $\left|\mathcal{X}_{p^{\prime}}\right|=40$. The best method in terms of the mean error and comparable methods according to the twosided paired t-test at the significance level $5 \%$ are specified by bold face. The standard deviation of the labeling error rate is given in brackets.

\begin{tabular}{l|cccccc}
\hline Dataset & DSDD & LSDD & KDE & KM & SC & SMIC \\
\hline australian & $\mathbf{. 2 4 4}(.116)$ & $.259(.088)$ & $.355(.104)$ & $.265(.080)$ & $.376(.065)$ & $.308(.107)$ \\
banana & $\mathbf{. 3 3 8}(.094)$ & $\mathbf{. 3 3 9}(.100)$ & $.365(.067)$ & $.433(.049)$ & $.427(.069)$ & $.424(.070)$ \\
diabetes & $\mathbf{. 3 4 0}(.075)$ & $.361(.124)$ & $.345(.034)$ & $.373(.063)$ & $.380(.048)$ & $.371(.114)$ \\
german & $.375(.042)$ & $.380(.093)$ & $\mathbf{. 3 5 4}(.057)$ & $.437(.024)$ & $.445(.057)$ & $.438(.041)$ \\
heart & $.270(.133)$ & $\mathbf{. 2 4 7}(.084)$ & $.354(.052)$ & $.264(.059)$ & $.315(.081)$ & $.327(.089)$ \\
image & $\mathbf{. 3 3 1}(.078)$ & $.350(.067)$ & $.350(.039)$ & $.384(.031)$ & $.354(.049)$ & $.382(.050)$ \\
ionosphere & $\mathbf{. 2 9 1}(.099)$ & $.356(.066)$ & $.345(.048)$ & $.330(.070)$ & $.322(.058)$ & $.314(.107)$ \\
saheart & $.378(.093)$ & $\mathbf{. 3 5 3}(.057)$ & $.363(.066)$ & $.419(.082)$ & $.395(.022)$ & $.385(.040)$ \\
thyroid &. $\mathbf{2 2 7}(.098)$ & $.251(.087)$ & $.302(.022)$ & $.326(.061)$ & $.329(.047)$ & $.307(.076)$ \\
twonorm & $.164(.188)$ & $.153(.121)$ & $.352(.096)$ & $\mathbf{. 0 3 6}(.053)$ & $.042(.122)$ & $.049(.120)$ \\
\hline
\end{tabular}

\section{Conclusion}

The problem of unsupervised labeling of two unbalanced datasets was considered. We first showed that this problem can be solved if two unlabeled datasets having different class balances are available. The solution can be obtained by estimating of the sign of the difference between probability densities. We introduced a method to directly estimate the sign of the density difference and avoid density estimation. The method was shown on various datasets to outperform 
competing methods that either estimate the density difference or use the cluster structure of the data.

Because the sign of density difference corresponds to the Bayes optimal classifier under equal class balance, it may be estimated by any classifier that separates $\mathcal{X}_{p}$ and $\mathcal{X}_{p^{\prime}}$. Following this idea, we tested the support vector machine (SVM) for estimating the sign of density difference. However, this did not work well due to the high overlap of $\mathcal{X}_{p}$ and $\mathcal{X}_{p^{\prime}}$ - both the datasets are mixtures of two classes, only with different mixing ratios.

From this classification point of view, we can actually see that our objective function (77) corresponds to the robust SVM [12] that minimizes the ramp loss (a clipped hinge loss). Thanks to the robustness brought by the ramp loss, the overlapped datasets $\mathcal{X}_{p}$ and $\mathcal{X}_{p^{\prime}}$ can be separated more reliably, and thus we obtained good estimation of the sign of density difference.

Furthermore, this view conversely shows that the robust SVM is actually a suitable classification method because it directly estimates the Bayes optimal classifier, the sign of density difference. Labeling and classification are different problems, but one can actually give insight into the other. In the future work, we will further investigate the relation between labeling and classification.

\section{References}

1. Vapnik, V.: The Nature of Statistical Learning Theory. Statistics for Engineering and Information Science Series. Springer (2000)

2. Sugiyama, M., Kanamori, T., Suzuki, T., du Plessis, M.C., Liu, S., Takeuchi, I.: Density-difference estimation. In Bartlett, P., Pereira, F., Burges, C., Bottou, L., Weinberger, K., eds.: Advances in Neural Information Processing Systems 25. (2012) 692-700

3. Silverman, B.: Density estimation for statistics and data analysis. Chapman and Hall, London, UK (1986)

4. Härdle, W., Müller, M., Sperlich, S., Werwatz, A.: Nonparametric and semiparametric models. Springer (2004)

5. Hall, P., Wand, M.P.: On nonparametric discrimination using density differences. Biometrika 75(3) (1988) 541-547

6. Anderson, N.H., Hall, P., Titterington, D.: Two-sample test statistics for measuring discrepancies between two multivariate probability density functions using kernelbased density estimates. Journal of Multivariate Analysis 50(1) (1994) 41-54

7. Yuille, A.L., Rangarajan, A.: The concave-convex procedure (CCCP). In: Advances in Neural Information Processing Systems 14, MIT Press (2002)

8. Horst, R., Thoai, N.V.: DC programming: overview. Journal of Optimization Theory and Applications 103(1) (1999) 1-43

9. Sugiyama, M., Kanamori, T., Suzuki, T., du Plessis, M.C., Liu, S., Takeuchi, I.: Density-difference estimation. Neural Computation (2013) to appear.

10. Shi, J., Malik, J.: Normalized cuts and image segmentation. Pattern Analysis and Machine Intelligence, IEEE Transactions on 22(8) (2000) 888-905

11. Sugiyama, M., Yamada, M., Kimura, M., Hachiya, H.: On informationmaximization clustering: Tuning parameter selection and analytic solution. In Getoor, L., Scheffer, T., eds.: Proceedings of 28th International Conference on Machine Learning (ICML2011), Bellevue, Washington, USA (Jun. 28-Jul. 2 2011) $65-72$ 
12. Shawe-Taylor, J., Cristianini, N.: Kernel Methods for Pattern Analysis. Cambridge University Press, New York, NY, USA (2004)

\section{A Optimization}

This section outlines the optimization of Eq. (7) using the convex concave procedure [7. The non-convex function $R(z)$ can be re-written as

$$
R(z)=C_{-1}(z)-C_{1}(z)-1, \text { where } C_{\epsilon}(z)=\max (0, z-\epsilon) .
$$

The convex part of the objective function can then be expressed as

$$
J_{\mathrm{vex}}(\boldsymbol{\alpha})=\frac{1}{n^{\prime}} \sum_{i=1}^{n^{\prime}} C_{-1}\left(\sum_{\ell=1}^{b} \alpha_{\ell} \varphi_{\ell}\left(\boldsymbol{x}_{i}^{\prime}\right)\right)+\frac{1}{n} \sum_{j=1}^{n} C_{1}\left(\sum_{\ell=1}^{b} \alpha_{\ell} \varphi_{\ell}\left(\boldsymbol{x}_{j}\right)\right)+\frac{\lambda}{2} \sum_{\ell=1}^{b} \alpha_{\ell}^{2},
$$

and the concave part as

$$
J_{\text {cave }}(\boldsymbol{\alpha})=-\frac{1}{n^{\prime}} \sum_{i=1}^{n^{\prime}} C_{1}\left(\sum_{\ell=1}^{b} \alpha_{\ell} \varphi_{\ell}\left(\boldsymbol{x}_{i}^{\prime}\right)\right)-\frac{1}{n} \sum_{j=1}^{n} C_{-1}\left(\sum_{\ell=1}^{b} \alpha_{\ell} \varphi_{\ell}\left(\boldsymbol{x}_{j}\right)\right) .
$$

The following self-evident relation can be used to bound the concave part

$$
\begin{gathered}
t z-\varphi(t) \leq \sup _{y \in \mathbb{R}} y z-\varphi(y) \\
\Rightarrow \varphi(t) \geq t z-\varphi^{*}(z),
\end{gathered}
$$

where

$$
\varphi^{*}(z)=\sup _{y \in \mathbb{R}} y z-\varphi(y)
$$

is known as the convex conjugate. The convex conjugate of the function $C_{\epsilon}(z)$ is

$$
C_{\epsilon}^{*}(z)= \begin{cases}\infty & z<0 \\ \epsilon z & 0 \leq z \leq 1 \\ \infty & z>0\end{cases}
$$

This gives an upper bound on the concave function as

$\bar{J}_{\text {cave }}(\boldsymbol{\alpha}, \boldsymbol{b}, \boldsymbol{c})=\frac{1}{n^{\prime}} \sum_{i=1}^{n^{\prime}}\left(C_{1}^{*}\left(b_{i}\right)-b_{i} \sum_{\ell=1}^{b} \alpha_{\ell} \varphi_{\ell}\left(\boldsymbol{x}_{i}^{\prime}\right)\right)+\frac{1}{n} \sum_{j=1}^{n}\left(C_{-1}^{*}\left(c_{j}\right)-c_{j} \sum_{\ell=1}^{b} \alpha_{\ell} \varphi_{\ell}\left(\boldsymbol{x}_{j}\right)\right)$,

where $\boldsymbol{b}=\left[\begin{array}{llll}b_{1} & b_{2} & \ldots & b_{n^{\prime}}\end{array}\right]$ and $\boldsymbol{c}=\left[\begin{array}{llll}c_{1} & c_{2} & \ldots & c_{n}\end{array}\right]$ specify the bound. 


\section{A.1 Tightening the bound}

The bound can be tightened around $\boldsymbol{\alpha}$ by minimizing $J_{\text {cave }}(\boldsymbol{\alpha}, \boldsymbol{b}, \boldsymbol{c})$ w.r.t. $\boldsymbol{b}$ and $\boldsymbol{c}$. To ensure that we have a non-trivial bound, we can explicitly write the conjugate as constraints,

$$
\begin{aligned}
\bar{J}_{\text {cave }}(\boldsymbol{\alpha}, \boldsymbol{b}, \boldsymbol{c})= & \frac{1}{n^{\prime}} \sum_{i=1}^{n^{\prime}} b_{i}\left(1-\sum_{\ell=1}^{b} \alpha_{\ell} \varphi_{\ell}\left(\boldsymbol{x}_{i}^{\prime}\right)\right)+\frac{1}{n} \sum_{j=1}^{n} c_{j}\left(-1-\sum_{\ell=1}^{b} \alpha_{\ell} \varphi_{\ell}\left(\boldsymbol{x}_{j}\right)\right) \\
& \text { s.t. } 0 \leq b_{i} \leq 1,0 \leq c_{j} \leq 1 .
\end{aligned}
$$

The above optimization problem is separable in all unknowns, and the optimal value can be obtained by Eq. (8).

\section{A.2 Minimizing the upper bound}

The upper bound of the objective function with $\boldsymbol{b}$ and $\boldsymbol{c}$ is

$$
J_{\mathrm{vex}}(\boldsymbol{\alpha})+\bar{J}_{\text {cave }}(\boldsymbol{\alpha}, \boldsymbol{b}, \boldsymbol{c}) .
$$

By replacing each function $C_{\epsilon}(z)$ with a slack variable $\xi_{i}$, and the constraint

$$
\xi_{i} \geq 0, \xi_{i} \geq z-\epsilon,
$$

we obtain the objective function in Eq. (9)

\section{B Least-squares estimation of the density difference}

In [9] it was proposed to directly estimate the density difference by fitting a model $g(\boldsymbol{x})$ to the true density difference $f(\boldsymbol{x})$ under a square loss:

$$
\underset{g}{\operatorname{argmin}} \frac{1}{2} \int\left(g(\boldsymbol{x})-\left[p(\boldsymbol{x})-p^{\prime}(\boldsymbol{x})\right]\right)^{2} \mathrm{~d} \boldsymbol{x} .
$$

The density difference was modeled by a linear-in-parameter model $g(\boldsymbol{x})$ :

$$
g(\boldsymbol{x})=\sum_{\ell=1}^{b} \theta_{\ell} \psi_{\ell}(\boldsymbol{x})=\boldsymbol{\theta}^{\top} \boldsymbol{\psi}(\boldsymbol{x})
$$

where $b$ denotes the number of basis functions, $\boldsymbol{\psi}(\boldsymbol{x})=\left(\psi_{1}(\boldsymbol{x}), \ldots, \psi_{b}(\boldsymbol{x})\right)^{\top}$ is a $b$-dimensional basis function vector, $\boldsymbol{\theta}=\left(\theta_{1}, \ldots, \theta_{b}\right)^{\top}$ is a $b$-dimensional parameter vector, and ${ }^{\top}$ denotes the transpose. A Gaussian kernel model is used to model the density difference:

$$
g(\boldsymbol{x})=\sum_{\ell=1}^{n+n^{\prime}} \theta_{\ell} \exp \left(-\frac{\left\|\boldsymbol{x}-\boldsymbol{c}_{\ell}\right\|^{2}}{2 \sigma^{2}}\right),
$$


where $\left(\boldsymbol{c}_{1}, \ldots, \boldsymbol{c}_{n}, \boldsymbol{c}_{n+1}, \ldots, \boldsymbol{c}_{n+n^{\prime}}\right):=\left(\boldsymbol{x}_{1}, \ldots, \boldsymbol{x}_{n}, \boldsymbol{x}_{1}^{\prime}, \ldots, \boldsymbol{x}_{n^{\prime}}^{\prime}\right)$ are Gaussian kernel centers. For the model in Eq. (10), the optimal parameter $\boldsymbol{\theta}^{*}$ is given by

$$
\begin{aligned}
\boldsymbol{\theta}^{*} & :=\underset{\boldsymbol{\theta}}{\operatorname{argmin}} \frac{1}{2} \int\left(g(\boldsymbol{x})-\left[p(\boldsymbol{x})-p^{\prime}(\boldsymbol{x})\right]\right)^{2} \mathrm{~d} \boldsymbol{x} \\
& =\underset{\boldsymbol{\theta}}{\operatorname{argmin}}\left[\frac{1}{2} \int g(\boldsymbol{x})^{2} \mathrm{~d} \boldsymbol{x}-\int g(\boldsymbol{x})\left[p(\boldsymbol{x})-p^{\prime}(\boldsymbol{x})\right] \mathrm{d} \boldsymbol{x}\right] \\
& =\underset{\boldsymbol{\theta}}{\operatorname{argmin}}\left[\frac{1}{2} \boldsymbol{\theta}^{\top} \boldsymbol{H} \boldsymbol{\theta}-\boldsymbol{h}^{\top} \boldsymbol{\theta}\right] \\
& =\boldsymbol{H}^{-1} \boldsymbol{h},
\end{aligned}
$$

where $\boldsymbol{H}$ is the $b \times b$ matrix and $\boldsymbol{h}$ is the $b$-dimensional vector defined as

$$
\begin{aligned}
\boldsymbol{H} & :=\int \boldsymbol{\psi}(\boldsymbol{x}) \boldsymbol{\psi}(\boldsymbol{x})^{\top} \mathrm{d} \boldsymbol{x}, \\
\boldsymbol{h} & :=\int \boldsymbol{\psi}(\boldsymbol{x}) p(\boldsymbol{x}) \mathrm{d} \boldsymbol{x}-\int \boldsymbol{\psi}(\boldsymbol{x}) p^{\prime}(\boldsymbol{x}) \mathrm{d} \boldsymbol{x} .
\end{aligned}
$$

For the Gaussian kernel model, the integral in $\boldsymbol{H}$ can be computed analytically as

$$
\begin{aligned}
H_{\ell, \ell^{\prime}} & =\int \exp \left(-\frac{\left\|\boldsymbol{x}-\boldsymbol{c}_{\ell}\right\|^{2}}{2 \sigma^{2}}\right) \exp \left(-\frac{\left\|\boldsymbol{x}-\boldsymbol{c}_{\ell^{\prime}}\right\|^{2}}{2 \sigma^{2}}\right) \mathrm{d} \boldsymbol{x} \\
& =\left(\pi \sigma^{2}\right)^{d / 2} \exp \left(-\frac{\left\|\boldsymbol{c}_{\ell}-\boldsymbol{c}_{\ell^{\prime}}\right\|^{2}}{4 \sigma^{2}}\right),
\end{aligned}
$$

where $d$ is the dimensionality of $\boldsymbol{x}$.

Replacing the expectations in $\boldsymbol{h}$ by empirical estimators and adding an $\ell_{2}$ regularizer to the objective function, we arrive at the following optimization problem:

$$
\widehat{\boldsymbol{\theta}}:=\underset{\boldsymbol{\theta}}{\operatorname{argmin}}\left[\frac{1}{2} \boldsymbol{\theta}^{\top} \boldsymbol{H} \boldsymbol{\theta}-\widehat{\boldsymbol{h}}^{\top} \boldsymbol{\theta}+\frac{1}{2} \lambda \boldsymbol{\theta}^{\top} \boldsymbol{\theta}\right],
$$

where $\lambda(\geq 0)$ is the regularization parameter and $\widehat{\boldsymbol{h}}$ is the $b$-dimensional vector defined as

$$
\widehat{\boldsymbol{h}}=\frac{1}{n} \sum_{i=1}^{n} \boldsymbol{\psi}\left(\boldsymbol{x}_{i}\right)-\frac{1}{n^{\prime}} \sum_{j=1}^{n^{\prime}} \boldsymbol{\psi}\left(\boldsymbol{x}_{j}^{\prime}\right) .
$$

Taking the derivative of the objective function in Eq. (11) and equating it to zero, we can obtain the solution $\widehat{\boldsymbol{\theta}}$ analytically as

$$
\widehat{\boldsymbol{\theta}}=\left(\boldsymbol{H}+\lambda \boldsymbol{I}_{b}\right)^{-1} \widehat{\boldsymbol{h}},
$$

where $\boldsymbol{I}_{b}$ denotes the $b$-dimensional identity matrix. Finally, the density difference estimator is

$$
\widehat{f}(\boldsymbol{x})=\widehat{\boldsymbol{\theta}}^{\top} \boldsymbol{\psi}(\boldsymbol{x}) .
$$

